\title{
CO Desorption from Oxygen Species on Carbonaceous Surface: 1. Effects of the Local Structure of the Active Site and the Surface Coverage
}

\author{
Alejandro Montoya, ${ }^{\dagger}$ Thanh-Thai T. Truong, ${ }^{\ddagger}$ Fanor Mondragón, ${ }^{*}, \dagger$ and Thanh N. Truong*, \\ Department of Chemistry, University of Antioquia, Medellin, Colombia A.A. 1226, Henry Eyring Center for \\ Theoretical Chemistry, Department of Chemistry, University of Utah, 315 South 1400 East, rm 2020, \\ Salt Lake City, Utah 84112
}

Received: February 14, 2001; In Final Form: May 1, 2001

\begin{abstract}
A systematic theoretical study using the density functional theory is performed to provide molecular-level understanding on the desorption of carbon monoxide from surface oxygen complexes that are formed in the gasification and combustion of coal. Particularly, a CO molecule release from carbonyl oxygen complexes in the presence of different oxygen environments was analyzed. Molecular carbonyl models of different sizes in the zigzag, armchair, and tip shapes of the active sites were selected. It was found that the shape of the local active site has a strong effect on the $\mathrm{CO}$ desorption energy, and they are correlated with the broaden feature of the $\mathrm{CO}$ molecule desorption in the temperature-programmed desorption (TPD) experiments of oxidized carbonaceous material. The calculated desorption activation energy range is in good agreement with experimental data. Molecular size convergence analyses on the carbonyl models suggest that the smallest graphene molecular system for accurate desorption structure on char is a three-ring molecule. The activation energy and normal-mode analyses for selected carbonyl complexes suggest that carbonyl surface oxygen complexes are stable structures and that they can be considered as labile surface oxygen complexes. The CO molecule desorption energy is affected by the influence of different neighboring surface oxygen groups on the carbon surface as well as the aromatic character of the molecular models. The desorption energy analysis suggests that the $\mathrm{CO}$ molecule can be adsorbed on clean carbonized surface to form stable structures. The preadsorbed oxygen group decreases the $\mathrm{CO}$ adsorption sticking probability on oxidized materials, but it remains an energetically favorable process.
\end{abstract}

\section{Introduction}

Understanding the reactivity of chars and carbons in reactions with oxygenated agents in the combustion and gasification processes has been the focus of many experimental studies due to its practical applications in industry. To improve the efficiency and to reduce the environmental impact of these processes, it is required to have knowledge of molecular-level detailed mechanisms of adsorption and desorption of different gases from the solid carbon or char surfaces. Experiments have addressed many questions related to the mechanism of reactions, but the complexity of the phenomena, however, has left many more challenging issues to be resolved. These issues require knowledge of microscopic properties that are not easily accessible by experimental methods such as adsorption sites, intermediates, inhibition of reactions, surface reconstruction after the desorption process, etc. Consequently, quantum mechanical methods have shown to be a viable alternative to experiments in enhancing our understanding for the mechanisms of this complex process at the molecular level. ${ }^{1-4}$

In general, the gasification process involves first the chemisorption of oxygen on the carbonaceous surface and subsequently desorption of carbon oxides upon heating. There have been a number of experimental studies on the mechanism of gasification, ${ }^{5-12}$ and a simplified reaction mechanism of a char with $\mathrm{CO}_{2}$ is shown in reactions $\mathrm{R} 1$ and $\mathrm{R} 2$ below $^{5}$

* Corresponding authors.

$\dagger$ University of Antioquia.

$\doteqdot$ University of Utah.

$$
\begin{gathered}
\mathrm{CO}_{2}+\mathrm{C}_{\mathrm{f}} \underset{k_{-1}}{\stackrel{k_{1}}{\rightleftarrows}} \mathrm{C}(\mathrm{O})+\mathrm{CO} \\
\mathrm{C}(\mathrm{O}) \stackrel{k_{2}}{\longrightarrow} \mathrm{C}_{\mathrm{f}}+\mathrm{CO}
\end{gathered}
$$

This mechanism assumes that certain carbon atoms from the char structure can chemisorb an oxygen atom from the $\mathrm{CO}_{2}$ to form a surface oxygen complex $\mathrm{C}(\mathrm{O})$ and to release a gas phase $\mathrm{CO}$ molecule. The surface oxygen complex subsequently can be released as a gas-phase $\mathrm{CO}$ molecule. It has been observed that the rate-determining step in the gasification by either $\mathrm{CO}_{2}$ or $\mathrm{O}_{2}$ is the $\mathrm{CO}$ release and the formation of a new active site on the carbonaceous solid surface, namely, the reaction R2. ${ }^{13,14}$ Thus, the nature of the active sites might affect the $\mathrm{CO}$ molecule desorption in the gasification and combustion of coal. Our interest in this paper is to analyze the heterogeneous surface of the char surface by analyzing the effect of the active sites, namely, zigzag, armchair, and zigzag shapes on the desorption of $\mathrm{CO}$ molecule from the surface oxygen complexes shown in the reaction $\mathrm{R} 2$. Chemisorption of $\mathrm{CO}_{2}$ on char will be presented in a separate report.

There have been numerous experimental efforts to identify surface oxygen complexes formed on carbon surfaces. The high temperatures at which the gasification and combustion take place make it difficult for experimental techniques to identify them in situ. Nevertheless, indirect techniques have been used to overcome the experimental difficulties in identifying surface 
oxygen complexes. For instance, studies using transient kinetic experiments have shown that in the char $-\mathrm{CO}_{2}$ and char $-\mathrm{O}_{2}$ reactions, there must be at least two kinds of surface oxygen complexes due to the desorption trend in the $\mathrm{CO}$ evolution which cannot be described by a single-exponential curve..$^{15-19}$ Several experimental studies have investigated the relative stability of these surface complexes. For instance, temperature-programmed desorption (TPD) spectra of surface complexes from oxidized carbons and chars have shown different kinds of surface oxygen complexes, depending on the temperature at which they are desorbed. ${ }^{20,21}$ In particular, TPD spectra obtained by Marchon et al. ${ }^{7}$ from disperse polycrystalline graphite oxidized at low temperature with $\mathrm{CO}$ show two main broad $\mathrm{CO}$ evolution features. One between 973 and $1253 \mathrm{~K}$ with a desorption energy in the range of $64-83 \mathrm{kcal} / \mathrm{mol}$ was assigned to semiquinone groups. Another $\mathrm{CO}$ evolution between 400 and $700 \mathrm{~K}$ with a desorption energy in the range of $25-44 \mathrm{kcal} / \mathrm{mol}$ was assigned to carbonyl groups. Although no direct identification of the surface oxygen complexes was done, Brown et al. ${ }^{22}$ have also observed the broadened evolution of $\mathrm{CO}$ at low temperature (400-600K) from oxidized carbon materials. It was suggested that the broadened TPD feature is due to the surface heterogeneity of the char. Other studies have also pointed out that carbonyl species can be formed not only at low temperatures after adsorption of $\mathrm{CO}$ but also at the gasification temperatures. ${ }^{12,23}$ Semiquinone and ether groups have been also proposed to account for the $\mathrm{CO}$ molecule desorption at high temperatures. ${ }^{24}$ These studies provided no direct information on the molecular structures of the surface oxygen species or the local nature of the char active site and how it affects the CO desorption property.

From the above discussion, it is clear that $\mathrm{CO}$ desorption from surface oxygen complexes, particularly from the carbonyl and semiquinone structures, is important to carbon gasification and combustion. To the best of our knowledge, there has not been yet any theoretical study on the $\mathrm{CO}$ desorption process from carbonyl-type surface oxygen species. Our goal in this study is to employ a state-of-the-art quantum chemistry method to address several fundamental questions regarding the desorption of carbonyl-type surface oxygen species as shown in the reaction R2. In particular, we examined (1) molecular structures, the stability of the carbonyl surface oxygen complexes, and the activation energy for $\mathrm{CO}$ desorption from such complexes, (2) the effects of the surface inhomogeneity due to differences in the local active site structures such as armchair, zigzag, and tip shapes and how they correspond to the usually broaden TPD peak obtained experimentally, and (3) the effects of neighboring surface oxygen complexes on the calculated $\mathrm{CO}$ desorption properties. The results from this study would help to validate the assignment of the surface oxygen complex that generates the TPD feature at low temperatures.

\section{Computational Details}

Physical Models. Henceforth, we differentiate the carbonyl group, $>=\mathrm{C}=\mathrm{O}$, where the carbon atom of $\mathrm{CO}$ group has the ketene structure and is not part of the six-member ring system from the ketone group, $>=\mathrm{O}$, where the carbon atom of the $\mathrm{C}=\mathrm{O}$ group belongs to the six-member ring system and is usually referred in the literature as semiquinone. ${ }^{25}$ In this study, the carbonyl models are represented by finite clusters of a single graphene layer where the edge atoms on the upper side are unsaturated to simulate the free active sites while others are terminated with hydrogen atoms as proposed by Radovic et al. ${ }^{26}$ and employed previously by Chen and Yang. ${ }^{27}$ Different models using different sizes and shapes that can be found in a carbonaceous structure were proposed, and they are presented in Figure 1. The first set of models, namely, z1/c, z3/c, z5/c, $\mathbf{z 6} / \mathbf{c}, \mathbf{z 7 / c}$, and $\mathbf{z 9 / \mathbf { c }}$, represents carbonyl groups in zigzag-shape char models of different sizes ( $\mathbf{z n} / \mathbf{c}$ denotes the zigzag-shape char model that has $\mathbf{n}$ six-member rings with a carbonyl group). In a similar notation, the second set of models, namely, t3/c and $\mathbf{t} 4 / \mathbf{c}$, represents carbonyl groups in the tip-shape char models, and the third set of models, namely, a4/c and a6/c, represents carbonyl groups in the armchair-shape char models.

Note that although char has a much larger structure where the graphene layers with various sizes have random orientations and translations, models considered in this study represent different local environments of the active site. The effects of the boundary conditions can be deduced from examining the convergence of the calculated physical properties with respect to the size of the model. The size of the models ranges from $\mathrm{C}_{7} \mathrm{H}_{5} \mathrm{O}$ to $\mathrm{C}_{32} \mathrm{H}_{10} \mathrm{O}$. They contain from one to three rows of six-member rings, where some of them are symmetric and others are not symmetric, that represent a subset of configurations that are important in chars. In Figure 1, some carbon atoms of the models are named to facilitate the discussion of the results below.

Theoretical Model. To calculate $\mathrm{CO}$ desorption activation energies from the carbonyl surface oxygen complexes, we fully optimized char models and their corresponding carbonyl surface oxygen complexes. Normal-mode analyses were performed to confirm their stability and to provide some information on the IR spectra of these surface complexes. All calculations were done at the B3LYP DFT level of theory, i.e., Becke's hybrid 3-parameter nonlocal exchange functional ${ }^{28}$ with Lee, Yang, and Parr's nonlocal correlation functional, ${ }^{29}$ using the $6-31 \mathrm{G}(\mathrm{d})$ basis set. It is known that B3LYP produces fairly accurate bond energies and thermodynamic properties of reactions and that the basis set used is adequate. Unrestricted open-shell wave function was used in all open-shell cases. In our previous study, we have shown that the spin contamination in the unrestricted B3LYP wave function is reasonably small and has acceptable small effects on the energetic properties. ${ }^{30}$ In addition, for each model, the ground electronic state was selected. This is done by performing single-point energy calculations at the same level of theory for different electronic states; the ground state is the lowest-energy one. All calculations were done using the GAUSSIAN 98 program. $^{31}$

\section{Results}

Carbonyl Surface Complex Models. The carbonyl models selected above were optimized in their ground state, and they are shown in Figure 1. All geometrical parameters in all models were fully optimized except for the carbonyl bond angle ie$\mathrm{C}-\mathrm{O}$ in the zigzag models. This angle was frozen at $180^{\circ}$ to facilitate the optimization. Normal-mode analyses on several zigzag carbonyl models confirmed that the optimized complexes are stable species. However, we found that complexes with similar structure on the armchair- and tip-shape models are not stable. The stable carbonyl-type surface oxygen complexes on the tip- and armchair-shape char models shows the $\mathrm{C}=\mathrm{O}$ group bonding with the two adjacent active site carbons to form a three-member ring. Note that even in this case, we still refer to it as the carbonyl group rather than the semiquinone group because the $\mathrm{C}=\mathrm{O}$ group is outside of the six-member ring structure of the graphene layer.

All carbonyl surface complex models having planar structure, selected geometrical parameters are summarized in Table 1. To 


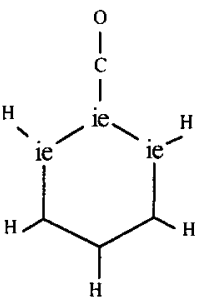

z1/c

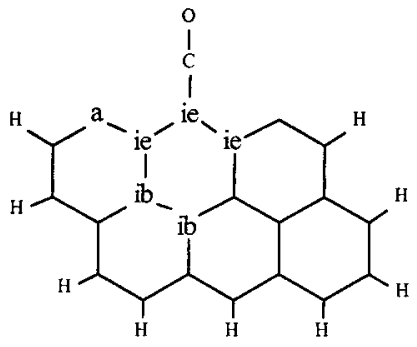

z6/c

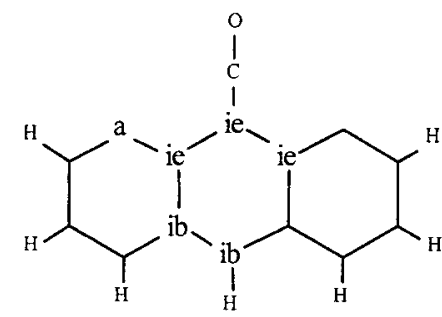

z3/c

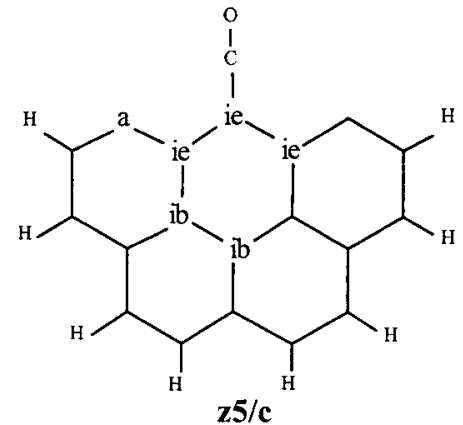

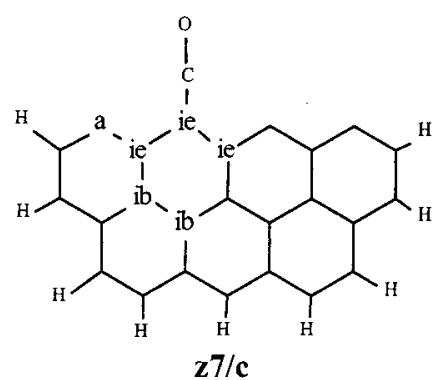

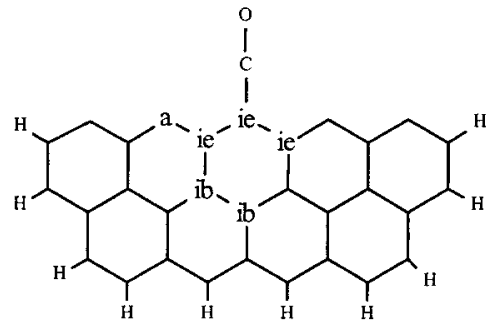

z9/c

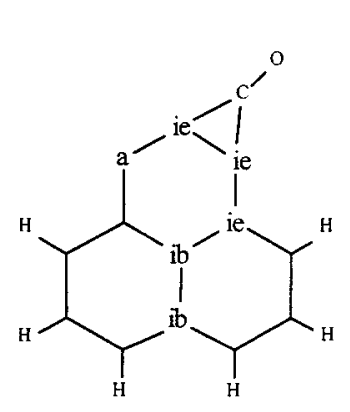

t3/c

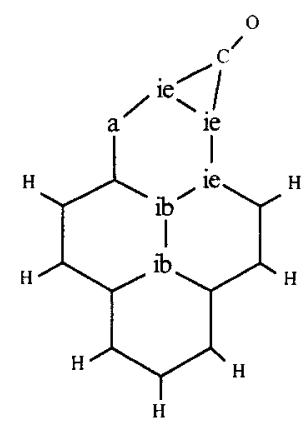

t4/c

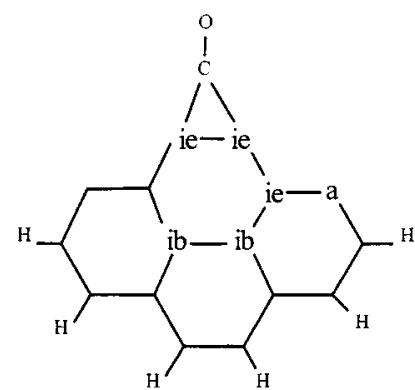

a4/c

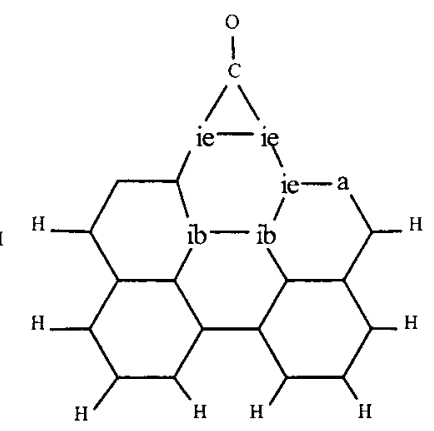

a6/c

Figure 1. Geometry representation of selected carbonyl models on a single graphene layer. Carbons are named in order to facilitate the discussion: (a) active, (ie) inactive exposed, and (ib) inactive buried.

TABLE 1: Average Values of Selected Optimized Geometrical Parameters of Unique Bonds and Angles for the Carbonyl Models ${ }^{a}$

\begin{tabular}{lccc}
\hline & \multicolumn{3}{c}{ carbonyl model type } \\
\cline { 2 - 4 } bond type & zigzag & tip & armchair \\
\hline $\mathrm{C}-\mathrm{O}$ & 116.8 & 120.6 & 120.4 \\
$\mathrm{C}-\mathrm{ie}$ & 134.4 & 144.9 & 144.2 \\
$\mathrm{ie}-\mathrm{ib}$ & 144.5 & 143.4 & 144.2 \\
$\mathrm{ib}-\mathrm{ib}$ & 142.3 & 142.8 & 144.9 \\
$\mathrm{a}-\mathrm{ie}$ & 139.4 & 136.6 & 139.0 \\
$\mathrm{ie}-\mathrm{ie}$ & 141.2 & 141.0 & 139.5 \\
$\mathrm{ie}-\mathrm{ie}-\mathrm{ie}$ & 120.6 & 123.9 & 124.0 \\
$\mathrm{C}-\mathrm{ie}-\mathrm{ie}$ & 119.9 & $61.4^{b}$ & $61.6^{b}$
\end{tabular}

${ }^{a}$ Bond lengths in pm and bond angles in deg. ${ }^{b}$ It corresponds to the three-member ring.

observe any trends or structure-function relationships from the large number of geometrical parameters of the char models, we first identified three unique carbon centers distinguished by their topology and then listed the average bond lengths formed by these centers in Table 1. The three unique carbon centers are the active, inactive exposed, and inactive buried carbon centers, as indicated by the letters "a", "ie", and "ib", respectively, in Figure 1. The active carbon represents the active site of char. It has no hydrogen atom termination and has a two-edge vertex. Inactive exposed carbon has a three-edge vertex. Inactive buried carbon atom also has three-edge vertex but differs from the ie carbon in that it does not exposed to the plane of adsorption of a gas-phase molecule, though this distinction is not always obvious in all cases. Thus, we will discuss only qualitatively the differences between ie and ib centers. Hydrogen atoms that are "capped" atoms are not important in this discussion.

First of all, we found that the average bond length of the $\mathrm{C}-\mathrm{O}$ group varies depending on the shape of the char model. Models in zigzag have the shortest average $\mathrm{C}-\mathrm{O}$ bond length, while models in tip and armchair shape have the longest average $\mathrm{C}-\mathrm{O}$ bond. Compared to the experimental value $\mathrm{e}^{32}$ of $112.8 \mathrm{pm}$ for an isolated carbon monoxide, the carbonyl $\mathrm{C}-\mathrm{O}$ bond in the zigzag models is the least affected one upon adsorption, followed by the carbonyl in tip and armchair models. Note that the adsorbed $\mathrm{C}$-ie bond lengths show the same trend, with those in the zigzag models having the smallest value, followed by those in the tip and armchair models. Since $\mathrm{CO}$ has a bidentate three-center bond to the tip and armchair model, one can expect the corresponding $\mathrm{C}-$ ie bond lengths to be longer than those of the other shape models that have the typical two-center bonds with the $\mathrm{C}=\mathrm{O}$ group. The others parameters correspond to the carbon matrix of the carbonyl models. By arranging them according to the bond type formed by the a, ie, and ib carbon centers, we can observe an important trend by in large in the bond length, depending mainly on its type rather than on its 


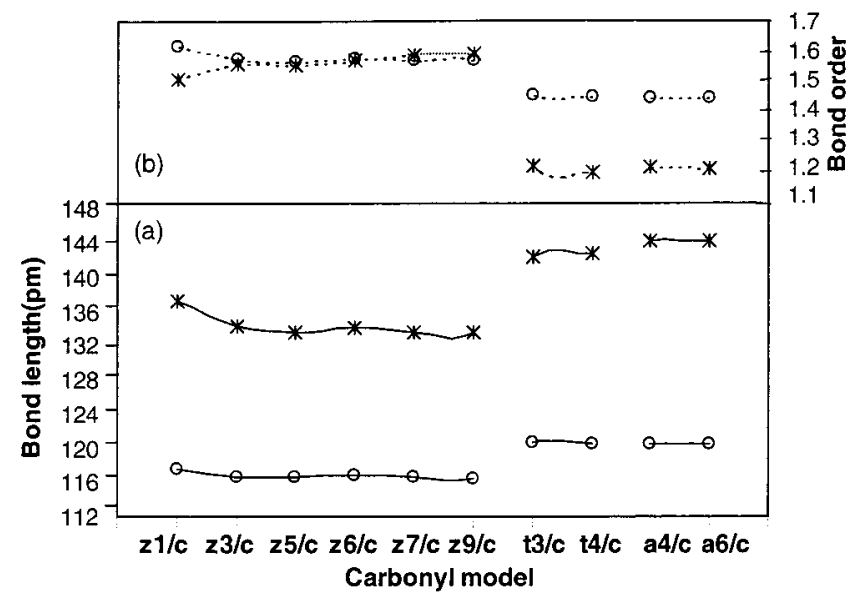

Figure 2. Change on selected optimized bond lengths in pm (a) and bond order analysis (AIM) (b) with the molecular size of the carbonyl models. (o) $\mathrm{C}-\mathrm{O}$ bond. (*) ie-C bond.

TABLE 2: Average Values of Selected Optimized Geometrical Parameters of Unique Bonds and Angles for the Carbon Models ${ }^{a}$

\begin{tabular}{lccc}
\hline & \multicolumn{3}{c}{ carbon model type } \\
\cline { 2 - 4 } bond type & zigzag & tip & armchair \\
\hline $\mathrm{a}-\mathrm{ie}$ & 139.1 & 141.7 & 139.0 \\
$\mathrm{ie}-\mathrm{ib}$ & 144.4 & 145.2 & 145.6 \\
$\mathrm{ib}-\mathrm{ib}$ & 141.9 & 142.9 & 144.0 \\
$\mathrm{a}-\mathrm{a}$ & & 129.5 & 123.7 \\
$\mathrm{a}-\mathrm{ie}$ & 126.6 & & 140.8 \\
ie $-\mathrm{a}-\mathrm{ie}$ & & 132.6 & \\
$\mathrm{a}-\mathrm{a}-\mathrm{a}$ & & & \\
ie $-\mathrm{a}-\mathrm{a}$ & & &
\end{tabular}

${ }^{a}$ Bond lengths in pm and bond angles in deg.

model shape. For instance, inactive-inactive bonds are longer than active-inactive bonds by more than $3 \mathrm{pm}$.

To investigate the dependence on the size of the models, we plot the two most important geometrical parameters associated with the $\mathrm{CO}$ desorption, namely, the $\mathrm{C}-\mathrm{O}$ and $\mathrm{C}-\mathrm{C}_{\mathrm{ie}}$ bonds, and their corresponding bond order determined by the theory of atoms in molecules ${ }^{33}$ as functions of model size as shown in panels a and b, respectively, of Figure 2. The values of the bond lengths and bond orders are connected for models of the same shape. As can be seen, with the exception of the benzene model (model $\mathbf{z 1 / c}$ ), the $\mathrm{CO}$ and $\mathrm{C}-\mathrm{C}_{\mathrm{ie}}$ bond lengths and bond orders converge with the size of the molecular model. This leads to an important conclusion: one requires at the smallest threering graphene molecular system to obtain accurate adsorption structure on char-type materials. Since carbonyl model z1/c is not adequate to model char, we will eliminate it from further discussion.

Carbon Models. We will refer to the carbon models as the carbonaceous product after the $\mathrm{CO}$ molecule is released from the carbonyl models shown in Figure 1. As nomenclature, the carbon model is denoted by the shape of the active site and the number of six-member rings; i.e., the carbonyl model " $\mathbf{z 3} / \mathbf{c}$ " produces the carbon model "z3" plus a CO molecule. These carbon models were fully optimized, and the similar statistical analysis data of their geometrical parameters are listed in Table 2. Note that upon the desorption of $\mathrm{CO}$, an active site is regenerated, and consequently, a new bond type, the a-a bond, appears in the tip-shape and armchair-shape models. By arranging according to the bond type as in Table 1, we obtained a similar conclusion. With the exception of the new a-a bond, other bond types do not have a strong dependence on the shape of the carbon model. The active-active $\mathrm{a}-\mathrm{a}$ bonds are noticeably shorter than the active-inactive bonds, which are in turn shorter than the inactive-inactive bonds. The short a-a bonds and the larger bond angles in the tip-shape and armchair-shape models indicate a significant bonding between the two neighboring singly occupied in-plane $\mathrm{sp}^{2}$ orbitals.

Table 3 depicts how the local structures of the carbon models change upon $\mathrm{CO}$ desorption, particularly the two nearest bonds to the carbonyl group. The closest $\mathrm{C}-\mathrm{C}$ bonds to the $\mathrm{C}-\mathrm{O}$ group will be referred as edge bonds. The distant $\mathrm{C}-\mathrm{C}$ bonds in the same ring will be referred as inner bonds. The geometrical changes in the nearest bond angles are also shown. Values in Table 3 were obtained from the differences between those of the carbon model and the carbonyl model. As can be seen, desorption of CO molecule yields only a small elongation in the inner bonds but a considerable contraction on the edge bonds and noticeable opening of the bond angles. This is indicated by small positive values of $\Delta L$ of the inner bonds in most cases and large negative values of $\Delta L$ of the edge bonds in all cases. The changes on the bond orders are also consistent with the observed changes in the structures. This finding suggests that desorption of $\mathrm{CO}$ molecule yields a considerable reorganization in the local structure of the active site to stabilize the newly generated lone-pair electron in the in-plane $\mathrm{sp}^{2}$ orbital.

CO Desorption Energies. The calculated desorption energies from different models are tabulated in Table 4. The $\mathrm{CO}$ desorption from a carbonyl model is an endothermic process without a transition state. We found that the $\mathrm{CO}$ desorption energy depends strongly on the shape of the local active site rather than on the size of the molecular model. In particular, variation in the $\mathrm{CO}$ desorption energy is small for different models having the same shape, whereas it is much larger for models with different shapes having the same size. For instance, the $\mathbf{z} 3 / \mathbf{c}$ and the $\mathbf{t} 3 / \mathbf{c}$ carbonyl models both consist of three sixmember rings but have the corresponding desorption energies of 47.0 and $33.0 \mathrm{kcal} / \mathrm{mol}$, respectively. Note the difference in the desorption energy from the two tip-shape models $\mathbf{t} 3 / \mathbf{c}$ and $\mathbf{t} 4 / \mathrm{c}$ is only $0.3 \mathrm{kcal} / \mathrm{mol}$. Thus, we can differentiate the desorption energy according to the model shape. Models in the

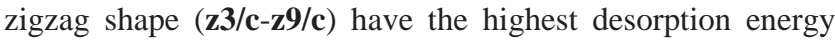
range from 40.2 to $54.9 \mathrm{kcal} / \mathrm{mol}$, whereas models in the tip shape have the lowest energy range from 33 to $33.3 \mathrm{kcal} / \mathrm{mol}$ and those in the armchair shape have a slightly higher range from 37.6 to $38.1 \mathrm{kcal} / \mathrm{mol}$. This is somewhat consistent with their bond orders shown in Figure 2.

One of the possibilities for the large dependence of the desorption energy on the shape of char model is the nature of the local environment of the active site. Notice that the zigzagshape models have one active site per active ring, whereas the armchair-shape models have two active sites per active ring and the tip-shape models have three active sites per active ring. Thus, the desorption energy decreases as the active site density increases. To further test this hypothesis, we calculated the CO desorption energy for a new carbonyl model a5/c, which has four active sites in the active ring as shown in Figure 3. In model $\mathbf{a 5} / \mathbf{c}$, the carbonyl group binds to the two active sites similar to the armchair models. We found that its desorption energy is $28.1 \mathrm{kcal} / \mathrm{mol}$, that is, $10 \mathrm{kcal} / \mathrm{mol}$ lower than that found in the armchair models, and thus it confirms the above observation.

It is interesting to note that unlike the tip-shape and armchairshape models, where the desorption energy shows a rather small variation of less than $3 \mathrm{kcal} / \mathrm{mol}$ in the model size, the zigzagshape models yield a rather large variation in the desorption energies of more than $14 \mathrm{kcal} / \mathrm{mol}$. Furthermore, the desorption 
TABLE 3: Difference in Some Optimized Selected Parameters of the Carbonyl and the Carbon Models ${ }^{a}$

\begin{tabular}{|c|c|c|c|c|c|c|c|c|c|c|c|}
\hline & & $\mathrm{z} 1 / \mathrm{c}$ & $\mathrm{z} 3 / \mathrm{c}$ & $\mathrm{z5} / \mathrm{c}$ & $\mathrm{z6} / \mathrm{c}$ & $\mathrm{z} 7 / \mathrm{c}$ & $\mathrm{z} 9 / \mathrm{c}$ & t3/c & $t 4 / c$ & a4/c & a6/c \\
\hline \multirow[t]{2}{*}{ edge } & $\Delta \mathrm{BL}$ & -6.6 & -6.2 & -7.6 & -6.9 & -7.3 & -5.4 & -11.0 & -11.83 & -13.0 & -13.2 \\
\hline & $\triangle \mathrm{BO}$ & 0.3 & 0.3 & 0.3 & 0.5 & 0.3 & 0.2 & 0.5 & 0.6 & 1.1 & 1.0 \\
\hline \multirow[t]{2}{*}{ inner } & $\Delta \mathrm{BL}$ & 3.2 & 0.2 & 2.2 & 1.8 & 2.4 & -0.4 & 0.7 & 0.4 & -1.3 & -1.5 \\
\hline & $\Delta \mathrm{BO}$ & -0.2 & -0.05 & -0.04 & -0.1 & -0.02 & 0.03 & -0.06 & -0.1 & 0.06 & 0.05 \\
\hline edge & $\triangle \mathrm{BA}$ & 7.0 & 6.7 & 5.8 & 5.9 & 5.6 & 4.1 & 8.3 & 8.4 & 3.9 & 3.8 \\
\hline
\end{tabular}

${ }^{a}$ Bond lengths in pm and bond angles in deg. $\Delta(x)=(x$ carbon model $-x$ carbonyl model $) . \mathrm{BL}=$ bond length. $\mathrm{BO}=$ bond order. $\mathrm{BA}=$ bond angle.

TABLE 4: Desorption Energy (kcal/mol) of the CO Molecule Release from the Carbonyl Models

\begin{tabular}{cccc}
\hline model & desorption energy & model & desorption energy \\
\hline $\mathbf{z 3} / \mathbf{c}$ & 47.0 & $\mathrm{t} 3 / \mathrm{c}$ & 33.0 \\
$\mathbf{z 5 / c}$ & 54.9 & $\mathrm{t} 4 / \mathrm{c}$ & 33.3 \\
$\mathbf{z 6} / \mathbf{c}$ & 40.2 & $\mathrm{a} 4 / \mathrm{c}$ & 38.1 \\
$\mathbf{z 7 / c}$ & 53.3 & $\mathrm{a} 6 / \mathrm{c}$ & 37.6 \\
$\mathbf{z 9 / c}$ & 54.8 & &
\end{tabular}

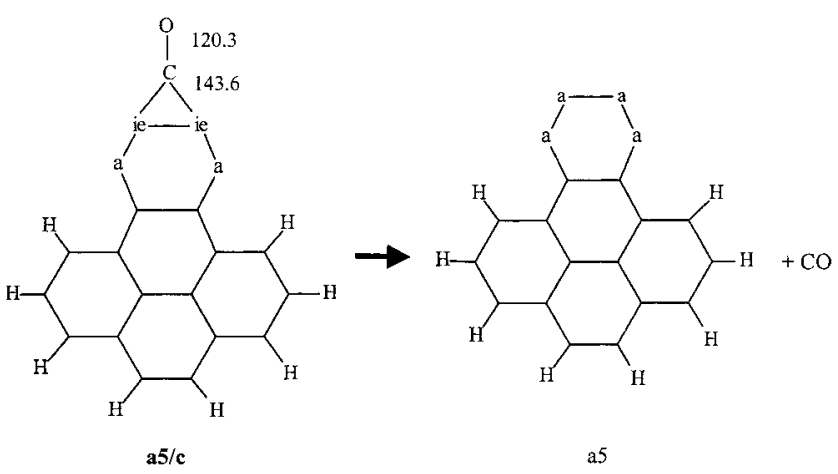

Figure 3. Geometry representations of the $\mathrm{CO}$ molecule desorption from the carbonyl model in armchair shape. The numbers represent selected optimized bond lengths in pm.

energies from the zigzag-shape models exhibit an oscillation behavior, with models $\mathbf{z 3 / \mathbf { c }}$ and $\mathbf{z 6 / \mathbf { c }}$ having the smaller values. This can be explained from the fact that among the carbon models $\mathbf{z 3}-\mathbf{z 9}$, only the $\mathbf{z 3}$ and $\mathbf{z 6}$ models are aromatic. Thus, $\mathbf{z 3}$ and $\mathbf{z 6}$ are more stable than other zigzag carbon models; hence, they yield much smaller desorption $\mathrm{CO}$ energies. In other words, resonance energy accounts for about $10-14 \mathrm{kcal} / \mathrm{mol}$.

To compare with experimental data, we need to add the zeropoint energy (ZPE) and thermal (temperature dependent) corrections to the calculated desorption energy. Thermal corrections are often small and can be neglected. We have used the carbonyl models $\mathbf{z 7 / c}, \mathbf{t} 4 / \mathbf{c}$, and $\mathbf{a} 4 / \mathbf{c}$ to estimate these zeropoint energy corrections for the zigzag-shape, tip-shape, and armchair-shape models, respectively. The ZPE corrections are estimated to be $3.9 \mathrm{kcal} / \mathrm{mol}$ for the zigzag models, $2.7 \mathrm{kcal} /$ mol for the tip models, and $3.8 \mathrm{kcal} / \mathrm{mol}$ for the armchair models. Hence, adding the ZPE corrections to the $\mathrm{CO}$ desorption energy in Table 1, we have predicted activation energy values from 31 to $49 \mathrm{kcal} / \mathrm{mol}$ for all models, in agreement with the Marchon's experimental value of CO desorption energy at low temperature. Also, the activation energy for the zigzag models are close to the experimental activation energy found by Fritz et al. ${ }^{20}$ They determined an experimental activation energy of $54.7 \mathrm{kcal} / \mathrm{mol}$ for complexes they called "labile". The present results suggest that one of the labile oxygen complexes in the gasification process is the carbonyl structure. The large spread on the activation energy of $\mathrm{CO}$ molecule desorption should broaden the $\mathrm{CO}$ desorption profile from oxidized char in a temperature-programmed desorption experiment. It is reasonable to suggest that the active sites in the zigzag-shape models are more likely the most abundant active sites in a char since such sites are more stable than those in the armchar- or tip-shape models due to the lower number of unsaturated carbon atoms in the six-member ring. The present energetic results suggest that the $\mathrm{CO}$ desorption profile in a TPD experiment will have a low shoulder at low temperatures and a higher signal intensity at higher desorption temperatures. Such a feature is in fact usually obtained experimentally.

To complete descriptions of the $\mathrm{CO}$ desorption from carbonyl surface complexes, one should also consider the steric effects from other graphene layers that could be found in a real carbonaceous matrix as well as the effects of neighboring oxygen complexes that are present in the gasification or combustion processes. The steric effect is difficult to address at the present time due to the computational time needed to study a more realistic model. The present results demonstrate that the $\mathrm{CO}$ desorption properties depend strongly on the local shape of the active site rather than the size of the molecular models, indicating the steric effects would be small. However, new computational approaches are being studied in our group that allow consideration of larger size molecular models. The influence of preadsorbed oxygen groups on the $\mathrm{CO}$ desorption energy will be address in the following section.

Effect of Neighboring $\mathrm{C}(\mathrm{O})$ Complexes on the $\mathrm{CO}$ Desorption. To study the effect of neighboring surface oxygen groups $\mathrm{C}(\mathrm{O})$ on the desorption of the $\mathrm{CO}$ molecule from the carbonyl model, we chose three different kind of reactions, as shown in Figure 4. Our goal here is to provide a qualitative understanding of such effects; thus, rigorous full optimizations and the dependence on the location of the neighboring group are not needed. In all reactions, model $\mathbf{z 7 / \mathbf { c }}$ was selected since it is large enough to place a neighbor surface oxygen group next to the carbonyl group and far enough from the model boundary. In Figure 4, reaction R1 is to model the effects of the neighboring carbonyl group. The reactant, model $\mathbf{z 7 / c c}$, and product were optimized with the constraints of the ie $-\mathrm{C}-\mathrm{O}$ carbonyl angles to be $180^{\circ}$. Reaction $\mathbf{R} 2$ is to model the effects of the neighboring semiquinone group. The semiquinone group has been extensively proposed and characterized by different experimental techniques as one of the most possible stable structures formed during the oxidation of the carbonaceous structures at the gasification temperatures. ${ }^{7,8,11,12,34-36}$ The

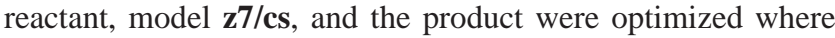
the ie- $\mathrm{C}-\mathrm{O}$ carbonyl angle , and the $\mathrm{ie}-\mathrm{ie}-\mathrm{O}$ semiquinone angles were frozen at $180^{\circ}$ and $120^{\circ}$, respectively. Reaction $\mathbf{R 3}$ is to model the effects of the epoxy oxygen group as in model z7/ce. It is important to mention that the epoxy group has not been confirmed experimentally; it was proposed from theoretical studies. ${ }^{1,2,37-39}$ We will use it as comparison with the previous surface oxygen complexes. During the optimization of the model $\mathbf{z 7 / c e}$, the ie $-\mathrm{C}-\mathrm{O}$ bond angle was frozen at $180^{\circ}$. The product of reaction after the $\mathrm{CO}$ desorption was fully optimized, and the epoxy oxygen group remained in the carbonaceous structure.

The geometry optimizations yield planar equilibrium structures for reactions $\mathbf{R} 1$ and $\mathbf{R} 2$ and nonplanar structures for the reaction $\mathbf{R} 3$. The epoxy oxygen group produces a deformation 


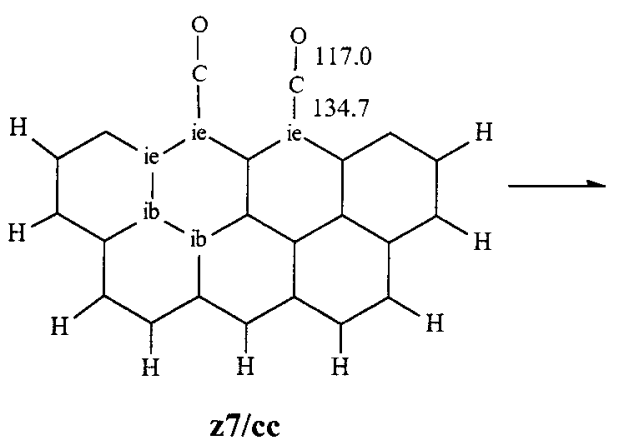

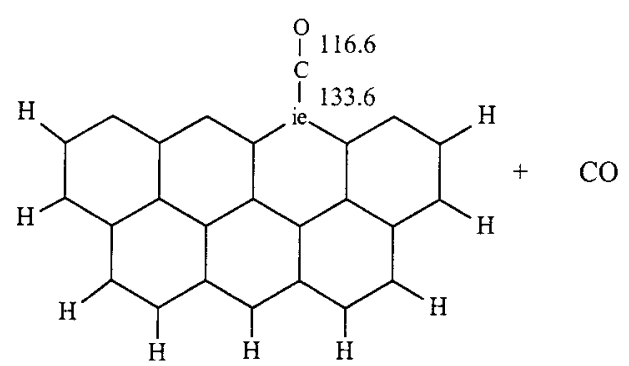

R1

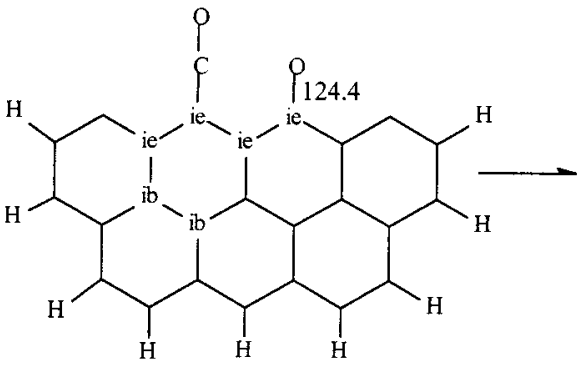

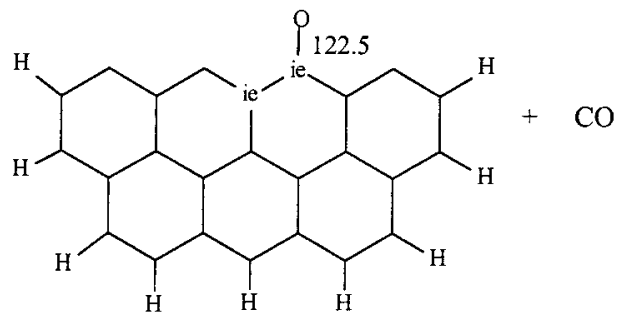

$\mathbf{2 7 / c s}$

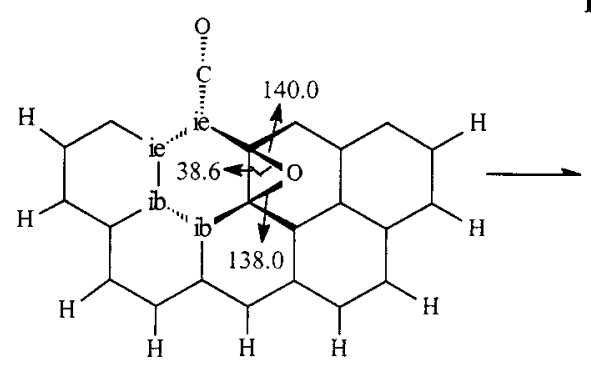

$\mathbf{R 2}$

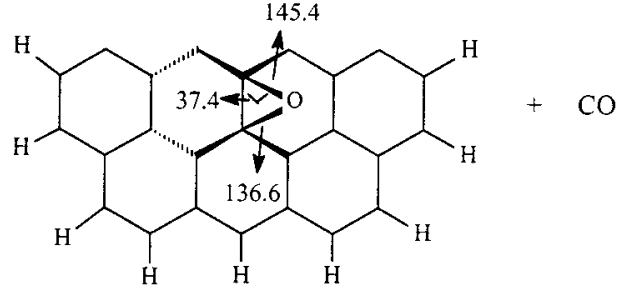

Z7/ce

\section{R3}

Figure 4. Geometry representations of the $\mathrm{CO}$ molecule desorption from carbonyl surface species in the presence of other oxygen groups. The numbers represent selected optimized parameters. Bond lengths in pm and bond angle in deg.

TABLE 5: Effect of Different Oxygen Complexes Near the Carbonyl C-O Group on the Desorption Energy (kcal/mol) of the CO Molecule Released

\begin{tabular}{lc}
\hline oxygen complex & desorption energy \\
\hline second carbonyl & 28.3 \\
semiquinone & 43.0 \\
epoxy & 10.3
\end{tabular}

of the planarity in the carbon model. The epoxy oxygen on both the reagent and in the product of reaction $\mathbf{R} \mathbf{3}$ was located over the carbon plane, as shown with bold lines in Figure 4. The carbonyl group was located below the carbon plane in the model z7/ce. Selected optimized geometrical parameters of the reactants and products of these reactions are also shown in Figure 4.

Table 5 lists the reaction energies of the reactions $\mathbf{R} \mathbf{1}-\mathbf{R} 3$, i.e., the $\mathrm{CO}$ desorption energy from models $\mathbf{z 7 / c c}, \mathbf{z 7} / \mathbf{c s}$, and $\mathbf{z 7 / c e}$, respectively. We found that the presence of the neighboring surface oxygen group decreases the $\mathrm{CO}$ desorption energy from the carbonyl group. The neighboring carbonyl group decreases the desorption energy by $46 \%$, while the semiquinone group decreases by $19.3 \%$. The largest effect is seen from the epoxy oxygen group: it decreases the $\mathrm{CO}$ desorption energy by $80.0 \%$. In this case, the desorption activation energy (with inclusion of the zero-point energy correction) is $8.1 \mathrm{kcal} / \mathrm{mol}$. This may be too low to be observed experimentally. Note that the epoxy oxygen group, if formed, can only be stabilized at very high temperatures due to the deformation of the planar graphene structure. This fact, combined with the very low $\mathrm{CO}$ desorption energy, makes it rather difficult to confirm the existence of such an epoxy group.

Identification of the Surface Oxygen Complexes. Fourier transform infrared spectra has been extensively used to identify surface oxygen complexes remaining on the surface after reacting carbon samples such us graphite, chars, or coals with oxidizing species. There is a concern in that carbonyl groups conjugated with the carbon matrix could be formed after oxidizing a carbon surface. Different peaks are usually found in the FTIR spectra on oxidized carbonized material, and they have been assigned to different groups. Peaks at 1840 and 1786 $\mathrm{cm}^{-1}$ have been assigned to the acid anhydride groups. ${ }^{40-42}$ Peaks at 1770-1710 have been usually identify as lactone group. ${ }^{34,43}$ Peaks in the range of $1710-1720 \mathrm{~cm}^{-1}$ have been attributed to the carboxyl or carbonyl group highly conjugated 
TABLE 6: Infrared Vibrational Frequencies $\left(\mathrm{cm}^{-1}\right)$ Predicted for Different Carbonyl Models

\begin{tabular}{lrcrr}
\hline vibrational mode & \multicolumn{1}{c}{$\mathrm{z} 7 / \mathrm{c}$} & $\mathrm{z} 7 / \mathrm{cs}$ & \multicolumn{1}{c}{$\mathrm{t} 4 / \mathrm{c}$} & \multicolumn{1}{c}{$\mathrm{a} 4 / \mathrm{c}$} \\
\hline $\mathrm{C}=\mathrm{C}$ stretching & $1420 / 3$ & $1525 / 2$ & $1429 / 2$ & $1434 / 3$ \\
& $1474 / 11$ & $1569 / 2$ & $1565 / 3$ & $1535 / 10$ \\
& $1609 / 6$ & & $1569 / 2$ & $1572 / 11$ \\
$\mathrm{C}=$ O stretching 177408n & $2144 / 100$ & $2099 / 100$ & $1869 / 100$ & $1887 / 100$ \\
page;2carbonyl & & & & \\
$\mathrm{C}=$ O stretching in & & $1584 / 8$ & & \\
semiquinone & & $1593 / 2$ & & \\
& & $1609 / 2$ & &
\end{tabular}

with the carbon surface. ${ }^{44}$ Peaks in the range of $1670-1610$ have also been considered as a measure of the amount of carbonyl groups highly conjugated with the carbon matrix. ${ }^{34,45}$ Table 6 shows the vibrational frequency predicted for selected carbonyl models. They were obtained at the same level of theory as that used for the optimization procedures. The B3LYP/6$31 \mathrm{G}(\mathrm{d})$ level is known to predict accurate vibrational frequencies, so any deviation from the experimental values will be analyzed in terms of the oxygen complexes formed during the gasification process rather than in the theoretical model. A scale factor of 0.9614 was used to convert the harmonic vibrational frequencies $(\omega)$ to the fundamentals frequencies $(v)$ observed experimentally. ${ }^{46}$ Table 6 shows the frequency and intensity values of the important vibrational modes. Model z7/c was selected to represent the carbonyl in zigzag shape, model t4/c to represent the carbonyl in tip shape, model a4/c to represent the carbonyl in arm-chair shape, and model $\mathbf{z 7 / c s}$ to represent the effect of the neighboring semiquinone group. This group of structures provides a rather complete picture of frequencies on carbonyl group in different local environments.

The frequencies predicted for the $\mathrm{C}=\mathrm{C}$ stretching in plane of the carbons in the member rings for all the carbonyl models in Table 6 are in the range of the experimental frequencies values found for the $\mathrm{C}=\mathrm{C}$ stretching in carbonaceous materials (1400 and $\left.1600 \mathrm{~cm}^{-1}\right){ }^{43}$ The $\mathrm{C}-\mathrm{O}$ stretching in the carbonyl group was predicted to be the most intense frequency in all cases. The $\mathrm{C}-\mathrm{O}$ frequency values for models $\mathbf{z 7 / \mathbf { c }}$ and $\mathbf{z 7 / \mathbf { c s }}$ are in the range of $2100-2150 \mathrm{~cm}^{-1}$. The ketone group does not affect the carbonyl mode of vibration. Infrared absorption bands at $2100 \mathrm{~cm}^{-1}$ have been assigned to the stretching in the $\mathrm{C} \equiv \mathrm{O}$ group on metals surfaces. ${ }^{47}$ These finding suggest a similar $\mathrm{C} \equiv \mathrm{O}$ character in the carbonyl models in zigzag-shape, as it is also suggested by their bond order (see Figure 2). The frequency values for the $\mathrm{C}-\mathrm{O}$ stretching in the semiquinone group in the $\mathbf{z 7 / c s}$ model is in agreement with the experimental values assigned to the $>\mathrm{C}=\mathrm{O}$ stretching mode. Models $\mathrm{t} 4 / \mathbf{c}$ and $\mathbf{a} 4 / \mathrm{c}$ have a $\mathrm{C}-\mathrm{O}$ absorption frequency in the range of 1870-1890 $\mathrm{cm}^{-1}$. This CO stretching frequency is different from the zigzag models because this group belongs to a three-member ring.

\section{Discussion}

Although the carbon models shown in Figure 1 do not represent a complete structure of a char, they can provide insights into the $\mathrm{CO}$ desorption pattern. The convergence with the molecular size of the geometrical parameters (Figure 2) and the desorption energy (Table 4) suggest that in much larger carbon models, the changes would be small, and the findings might be considered general. The linear $>\mathrm{C}=\mathrm{C}=\mathrm{O}$ carbonyl group have been proposed previously as a possible oxygen group formed on oxidized carbons. ${ }^{7}$ The normal-mode analysis on the zigzag shape carbonyl models confirmed that they are stable structures. To our knowledge, the three-member ring carbonyl group in the tip- and armchair-shape models has not been considered previously. The three-member ring is a stable structure and has noticeable positive $\mathrm{CO}$ binding energy; thus, it should be possible to observe such complexes experimentally. The $\mathrm{C}=\mathrm{O}$ frequency value in the three-member ring (Table 6) shows that it can be overlapped with the acid anhydride frequency signal in the region of $1800 \mathrm{~cm}^{-1}$.

The bond orders shown in Figure 2 suggest that the fission of the $\mathrm{Cie}-\mathrm{C}$ bond is easier than of the $\mathrm{C}-\mathrm{O}$ bond in the tip and armchair models. The $\mathrm{CO}$ evolution would be the preferred channel compared to breaking the $\mathrm{C}-\mathrm{O}$ bond and leaving the carbon atom of the $\mathrm{CO}$ group on the carbonaceous surface. In the zigzag-shape models, the $\mathrm{C}-\mathrm{Cie}$ and $\mathrm{C}-\mathrm{O}$ bond orders have similar magnitudes of about 1.56. This indicates that the carbonyl group is highly conjugate with the carbonaceous matrix. In addition, it suggests that there are two possible channels in the high temperature region, namely, the desorption of $\mathrm{CO}$ molecule and the oxygen migration of the linear $>\mathrm{C}=$ $\mathrm{C}=\mathrm{O}$ bond to a neighboring active site to form a cyclic ether group $>\mathrm{C}-\mathrm{C}-\mathrm{O}-\mathrm{C}<$ without breaking the $\mathrm{C}-\mathrm{O}$ bond. The highest-frequency peak usually obtained in the infrared spectra of oxidized carbonized materials is at about $1840 \mathrm{~cm}^{-1}$. Notice that the predicted carbonyl frequencies in the zigzag models are about $300 \mathrm{~cm}^{-1}$ higher than the largest experimental values. Although predicted frequency values can deviate about $200 \mathrm{~cm}^{-1}$ from the experimental observation, it suggests that if the carbonyl complex is formed during the gasification process, they can be transformed to a subsequent ether group. ${ }^{34,40-45}$ The adsorption of the $\mathrm{CO}$ molecule to form an ether group is currently being studied in our group.

The CO desorption activation energies listed in Table 4 suggest that the reverse reaction is a favorable process. The CO molecule can be adsorbed on clean carbonized surface to form stable carbonyl structures. From Table 5, the preadsorbed oxygen species decrease the adsorption sticking probability of the $\mathrm{CO}$ molecule on oxidized material, but it remains to be an energetically favorable process. The $\mathrm{CO}$ adsorption process decreases the number of active sites, whereas the $\mathrm{CO}$ desorption process from a carbonyl surface regenerates the active sites on the carbon substrate. The adsorption/desorption cycle from carbonyl surface species keeps the number of active sites constant during the gasification process. The low propensity of the carbonaceous surface to adsorb/(re)adsorb the $\mathrm{CO}$ molecule ${ }^{22}$ suggest that the desorption of carbon oxides from different oxygen complexes involves an irreversible rearrangement of the carbon surface after the desorption process and decreases the ability to adsorb $\mathrm{CO}$ molecule by changing the nature of the active site. On the other hand, as pointed out by Calo and Hall, ${ }^{24}$ the reactivity behavior of a $\mathrm{CO}$ gas molecule may depend on the local microporous structure of char. For instance, if a $\mathrm{CO}$ molecule is released in a narrow pore of char from any surface complex, it is possible that it can readsorb to form a new and different surface oxygen complex. Currently, the formation of different surface oxygen complexes is being studied in our group.

\section{Conclusions}

We have carried out a systematic theoretical study using a nonlocal hybrid B3LYP density functional theory to provide insight into the mechanism of $\mathrm{CO}$ molecule desorption from carbonyl surface species in the gasification and combustion of char.

Different local active sites of the carbonyl group were studied in order to establish the effect of surface heterogeneity on the $\mathrm{CO}$ molecule desorption energy. We found that the spread of 
the desorption activation energy depends not only on the shape of the active site but also on the aromatic character of the molecular model and the oxygen complex environment that surrounds the carbonyl group. Though contrary to the normal understanding, it does not depend strongly on the size of the models. However, two distinct ranges of $\mathrm{CO}$ desorption energy are predicted. Carbonyls in tip and armchair shapes have small desorption activation energy, and they will release first a $\mathrm{CO}$ gas molecule at low temperatures in a TPD experiment. Then, as the temperature increases, desorption of a $\mathrm{CO}$ gas molecule from zigzag carbonyl models follows. Our calculated desorption activation energy ranging from 31 to $49 \mathrm{kcal} / \mathrm{mol}$ is in good agreement with experimental data.

Note that carbonyl surface oxygen complex is not only limited to the $\mathrm{CO}$ molecule adsorption at low temperatures on the clean surface of a char but also they can be formed by the transformation of other surface oxygen complexes such us lactones, ethers, and acid anhydrides. Such transformations are important in a detailed mechanism of gasification and combustion reactions and should also be considered.

Acknowledgment. T. N. Truong acknowledges financial support from NSF. F. Mondragón and A. Montoya want to thank Colciencias and the University of Antioquia for financial support of Project 1115-05-10853. We also thank the Utah Center for High Performance computing for computer time support.

Note Added after ASAP Posting. This article was posted ASAP with duplicated references on 6/14/01. The correct version was posted on $7 / 5 / 01$.

\section{References and Notes}

(1) Chen, S. G.; Yang, R. T. Energy Fuels 1997, 11, 421.

(2) Chen, N.; Yang, R. T. J. Phys. Chem. A 1998, 102, 6348.

(3) Kyotani, T.; Tomita, A. J. Phys. Chem. B 1999, 103, 3434.

(4) Montoya, A.; Truong, A. N.; Sarofim, A. F. J. Phys. Chem A 2000, 104,8409 .

(5) Ergun, B. S. J. Phys. Chem. 1956, 60, 480.

(6) Mentser, M.; Ergun, S. Carbon 1967, 5, 331.

(7) Marchon, B.; Tysoe, W. T.; Carrazza, J.; Heinemann, H.; Somorjai, G. A. J. Phys. Chem. 1988, 92, 5744.

(8) Marchon, B.; Carrazza, J.; Heinemann, H.; Somorjai, G. A. Carbon 1988, 26, 507 .

(9) Lizzio, A. A.; Jiang, H.; Radovic, L. R. Carbon 1990, $28,7$.

(10) Radovic, L. R.; Jiang, H.; Lizzio, A. A. Energy Fuels 1991, 5, 68.

(11) Zhuang, Q.; Kyotani, T.; Tomita, A. Energy Fuels 1995, 9, 630.

(12) Moulijn, J. A.; Kapteijn, F. Carbon 1995, 33, 1155.

(13) Hüttinger, K. J.; Fritz, O. W. Carbon 1991, 29, 1113.

(14) Hüttinger, K. J.; Nill, J. S. Carbon 1990, 28, 457.

(15) Freund, H. Fuel 1986, 65, 63.

(16) Radovic, L. R.; Jiang, H.; Lizzio, A. A. Energy Fuels 1991, 5, 68.

(17) Kapteijn, F.; Meijer, R.; Moulijn, J. A. Energy Fuels 1992, 6, 494.
(18) Kapteijn, F.; Meijer, R.; Eck, B. V.; Moulijn, J. A. Fundamental Issues in Control of Carbon Gasification Reactivity; Kluwer Academic Publishers: Dordrecht, The Netherlands, 1991; p 221.

(19) Calo, J. M. Fundamental Issues in Control of Carbon Gasification Reactivity; Kluwer Academic Publishers: Dordrecht, The Netherlands, 1991; p 369.

(20) Fritz, O. W.; Hüttinger, K. J. Carbon 1993, 31, 923.

(21) Lizzio, A. A.; Radovic, L. R. Prepr. Pap.-Am. Chem. Soc., Div of Fuel Chem. 1989, 30 (1), 103.

(22) Brown, T. C.; Haynes, B. S. Energy Fuels 1992, 6, 154.

(23) Kapteijn, F.; Meijer, R.; Moulijn, J. A.; Cazorla-Amoros, D. Carbon 1994, 32, 1223 .

(24) Calo, J. M; Hall, P. J. Fundamental Issues in Control of Carbon Gasification Reactivity; Kluwer Acadmic Publisher: Dordrecht, The Netherlands, 1991; p 329.

(25) A Guide to IUPAC Nomenclature of Organic Compounds (Recommendations 1993); Rules C321.2 and R-5.6.2; Blackwell Scientific Publications: Cambridge, MA, 1993.

(26) Radovic, L. R.; Walker, P. L.; Jenkins, R. G. Fuel 1983, 62, 849.

(27) Chen, N.; Yang, R. T. Carbon 1998, 36, 1061.

(28) (a) Becke, A. D. J. Chem. Phys. 1992, 96, 2155. (b) Becke, A. D. J. Chem. Phys. 1992, 97, 9173. (c) Becke, A. D. J. Chem. Phys. 1993, 98 5648 .

(29) Lee, C.; Yang, W.; Parr, R. G. Phys. Rev. B. 1988, 37, 785.

(30) Montoya, A.; Truong, T.; Sarofim, A. F. J. Phys. Chem A 2000, 104, 6108 .

(31) Frisch, M. J.; Trucks, G. W.; Schlegel, H. B.; Scuseria, G. E.; Robb, M. A.; Cheeseman, J. R.; Zakrzewski, V. G.; Montgomery, J. A., Jr.; Stratmann, R. E.; Burant, J. C.; Dapprich, S.; Millam, J. M.; Daniels, A D.; Kudin, K. N.; Strain, M. C.; Farkas, O.; Tomasi, J.; Barone, V.; Cossi, M.; Cammi, R.; Mennucci, B.; Pomelli, C.; Adamo, C.; Clifford, S.; Ochterski, J.; Petersson, G. A.; Ayala, P. Y.; Cui, Q.; Morokuma, K.; Malick, D. K.; Rabuck, A. D.; Raghavachari, K.; Foresman, J. B.; Cioslowski, J.; Ortiz, J. V.; Baboul, A. G.; Stefanov, B. B.; Liu, G.; Liashenko, A.; Piskorz, P.; Komaromi, I.; Gomperts, R.; Martin, R. L.; Fox, D. J.; Keith, T.; AlLaham, M. A.; Peng, C. Y.; Nanayakkara, A.; Gonzalez, C.; Challacombe, M.; Gill, P. M. W.; Johnson, B.; Chen, W.; Wong, M. W.; Andres, J. L.; Gonzalez, C.; Head-Gordon, M.; Replogle, E. S.; Pople, J. A. Gaussian 98, Revision A.9; Gaussian, Inc.: Pittsburgh, PA, 1998.

(32) CRC Handbook of Chemistry and Physics, 81 st ed; CRC press: Cleveland, OH, 2000; pp 9-19.

(33) Bader, R. F. W. Atoms in Molecules: A Quantum Theory; Oxford University Press: Oxford, 1990.

(34) Zhuang, Q. L.; Kyotani, T.; Tomita, A. Energy Fuels 1994, 8, 714.

(35) Akhter, M. S.; Keifer, J. R.; Chughthai, A. R.; Smith, D. M. Carbon 1985, 23, 589.

(36) Tagahagi, T.; Ishitani, I. Carbon 1984, 22, 43.

(37) Pan, Z.; Yang, R. T. Ind. Eng. Chem. Res. 1992, 31, 2675.

(38) (a) Beran, S.; Dubsky, J.; Slanina, Z. Surf. Sci. 1979, 79, 39. (b) Dubsky, J.; Beran, S. Surf. Sci. 1979, 53.

(39) (a) Chen, S. G.; Yang, R. T. J. Catalysis 1993, 141, 102.

(40) (a) Meldrum, B. J.; Rochester, C. H. J. Chem. Soc., Faraday Trans. 1990, 86, 861. (b) 1990, 86, 1881.

(41) Meldrum, B. J.; Rochester, C. H. Fuel 1991, 70, 57.

(42) Fanning, P. E.; Vannice, M. A. Carbon 1993, 31, 721.

(43) Gómes-Serrano, V.; Piriz-Almeida, F.; Durán-Valle, C. J.; VillegasPastor, J. Carbon 1999, 37, 1517.

(44) Hontoria-Lucas, C.; López-Peinado, J. D.; Rojas-cervantes, M. L.; Martín-Aranda, R. M. Carbon 1995, 33, 1585.

(45) Shin, A.; Jang, J.; Yoon, S.-H.; Mochida, I. Carbon 1997, 35, 1739.

(46) Scott, A. P.; Radom, L. J. Phys. Chem. 1996, 100, 16502.

(47) Huhey, J. E. In Química Inorgánica. Principios de Estructura y Reactividad; México: Harla, 1981; pp 358-456. 